\title{
Robust Adaptive Exponential Synchronization of Stochastic Perturbed Chaotic Delayed Neural Networks with Parametric Uncertainties
}

\author{
Yang Fang, ${ }^{1}$ Kang Yan, ${ }^{1}$ and Kelin $\mathrm{Li}^{2}$ \\ ${ }^{1}$ School of Automation and Electronic Information, Sichuan University of Science \& Engineering, Sichuan 643000, China \\ ${ }^{2}$ Institute of Nonlinear Science and Engineering Computing, Sichuan University of Science \& Engineering, Sichuan 643000, China \\ Correspondence should be addressed to Kelin Li; 1kl@suse.edu.cn
}

Received 27 December 2013; Revised 23 May 2014; Accepted 23 May 2014; Published 23 June 2014

Academic Editor: Yang Tang

Copyright (C) 2014 Yang Fang et al. This is an open access article distributed under the Creative Commons Attribution License, which permits unrestricted use, distribution, and reproduction in any medium, provided the original work is properly cited.

\begin{abstract}
This paper investigates the robust adaptive exponential synchronization in mean square of stochastic perturbed chaotic delayed neural networks with nonidentical parametric uncertainties. A robust adaptive feedback controller is proposed based on Gronwally's inequality, drive-response concept, and adaptive feedback control technique with the update laws of nonidentical parametric uncertainties as well as linear matrix inequality (LMI) approach. The sufficient conditions for robust adaptive exponential synchronization in mean square of uncoupled uncertain stochastic chaotic delayed neural networks are derived in terms of linear matrix inequalities (LMIs). The effect of nonidentical uncertain parameter uncertainties is suppressed by the designed robust adaptive feedback controller rapidly. A numerical example is provided to validate the effectiveness of the proposed method.
\end{abstract}

\section{Introduction}

Synchronization of chaotic delayed neural networks has been an intensive topic because of its promising connections with many disciplines, such as image encryption [1], image processing [2], harmonic oscillation generation [3], and secure communications [4-6]. The diverse control schemes have been proposed for the synchronization of chaotic delayed neural networks, for example, adaptive control (see, [7-13]), slide mode control [14, 15], coupling control [16], feedback control [17-21], impulsive control [22-25], and sampled-data control [26-28].

The existence of random uncertainties such as stochastic noise in the electrical circuits design of neural networks possesses an important source in what may change or destroy the synchronization. Therefore, the stochastic effects must be taken into consideration for the synchronization problem of chaotic delayed neural networks. Some works on the synchronization of stochastic perturbed chaotic neural networks have been reported in the literature; see $[8,9,17$, $29,30]$ and the references therein. The authors in [29] were concerned with the problem of exponential synchronization for stochastic jumping chaotic neural networks (SJCNNs) with mixed delays and sector nonlinearities by employed Lyapunov-Krasovskii functional and free-weighting matrix method and proposed a delay-dependent feedback controller with sector nonlinearities to achieve the synchronization in mean square in terms of linear matrix inequalities (LMIs). In [9], Zhu and Cao have derived some novel sufficient conditions achieving complete synchronization of unidirectionally coupled stochastic delayed neural networks by utilizing LaSalle invariant principle of stochastic differential delay equations and the stochastic analysis as well as the adaptive feedback control technique and LMI approach. Li et al. in [30] investigated the synchronization problem of a class of chaotic neural networks with time-varying delays and unbounded distributed delays under stochastic perturbations via Lyapunov-Krasovskii functional, drive-response concept, output coupling with delay feedback, and LMI approach, some sufficient conditions in terms of LMIs ensuring the exponential synchronization of the addressed neural networks are derived.

On the other hand, besides stochastic noise, it is well known that the effects of parametric uncertainties which 
may also destroy the stability of the controlled system cannot be ignored in many applications. However, all of the abovementioned works mainly focus on the stochastic perturbed chaotic delayed neural networks without parametric uncertainties. According to the best of our knowledge, there are still few results about the synchronization of stochastic perturbed chaotic delayed neural networks with nonidentical parametric uncertainties. This is the motivation of our research in the present paper.

In this paper, the main aim is to design a robust adaptive feedback controller with the update laws of nonidentical parametric uncertainties and find some sufficient conditions in order to guarantee exponential synchronization in mean square for uncoupled chaotic delayed neural networks with stochastic perturbation and parametric uncertainties. Based on Gronwally's inequality, drive-response concept, adaptive feedback control technique, and linear matrix inequality (LMI) approach, several sufficient conditions in the form of linear matrix inequalities (LMIs) are derived to ensure exponential synchronization in mean square for uncoupled uncertain stochastic chaotic delayed neural networks. In addition, the existence of the desired controller can be validated by MATLAB LMI toolbox efficiently. The significant difference from previous results is that the nonidentical parametric uncertainties are entered into both the connection weight matrix and the delayed connection weight matrix in the drive-response systems. The task for compensating the nonidentical parametric uncertainties can be realized by the designed robust adaptive feedback controller rapidly. Moreover, we have pointed out that the LaSalle invariance principle for stochastic differential delay equation [31, Corollary 3.1] cannot be applied for the stability analysis of stochastic delayed systems without a trivial solution $e(t ; 0)=0$. Finally, a numerical example and its simulation are given to illustrate the usefulness of the given method.

Notation. Let $\mathbb{R}$ denote the set of real numbers, let $\mathbb{R}^{+}$denote the set of all nonnegative real numbers, and $\mathbb{R}^{n}$ and $\mathbb{R}^{n \times m}$ denote the $n$-dimensional and $n \times m$ dimensional real spaces equipped with the Euclidean norm; $\|z\|$ is the Euclidean norm of the vector $z$. $\mathbb{N}$ denotes the set of positive integers. For any matrix $X \in \mathbb{R}^{n \times n}, X>0$ denotes that $X$ is a symmetric and positive definite matrix. If $X_{1}$ and $X_{2}$ are symmetric matrices, then $X_{1} \leq X_{2}$ means that $X_{1}-X_{2}$ is a negative semidefinite matrix. $X^{T}$ and $X^{-1}$ mean the transpose of $X$ and the inverse of a square matrix. $I$ denotes the identity matrix with appropriate dimensions. Let $\tau>0$ and $C\left([-\tau, 0] ; \mathbb{R}^{n}\right)$ denote the family of all continuous $\mathbb{R}^{n}$-valued functions $\xi(\theta)$ on $[-\tau, 0]$ with the norm $\|\xi\|=\sup _{-\tau \leq \theta \leq 0}|\xi(\theta)|$. Let $C_{\mathscr{F}_{0}}^{b}\left([-\tau, 0] ; \mathbb{R}^{n}\right)(t \geq 0)$ denote the family of all $\mathscr{F}_{0}$ measurable bounded $C\left([-\tau, 0] ; \mathbb{R}^{n}\right)$-valued random variables $\xi=\{\xi(\theta):-\tau \leq \theta \leq 0\}$, such that $\int_{-\tau}^{0} E|\xi(s)|^{2} d s<\infty$, where $E\{\cdot\}$ stands for the correspondent expectation operator with respect to the given probability measure $\mathscr{P}$. Let $C^{1,2}\left(\mathbb{R}^{+} \times\right.$ $\left.\mathbb{R}^{n} ; \mathbb{R}^{+}\right)$denote the family of all nonnegative functions $V(t, x)$ on $\mathbb{R}^{+} \times \mathbb{R}^{n}$ which are continuously twice differentiable in $x$ and differentiable in $t$. The notation $\star$ always denotes the symmetric block in one symmetric matrix.

\section{Model Description and Preliminaries}

Consider the following chaotic delayed neural networks with time-varying parametric uncertainties:

$$
\begin{aligned}
d x(t)=[ & -C x(t)+\left(A+\Delta A_{1}(t)\right) f(x(t)) \\
& \left.+\left(B+\Delta B_{1}(t)\right) f(x(t-\tau(t)))+J\right] d t,
\end{aligned}
$$

where $x(t)=\left(x_{1}(t), x_{2}(t), \ldots, x_{n}(t)\right)^{T}$ is the neuron state vector, $C$ is a positive diagonal matrix, $A=\left(a_{i j}\right) \in \mathbb{R}^{n \times n}$ and $B=$ $\left(b_{i j}\right) \in \mathbb{R}^{n \times n}$ are the connection weight matrix and the delayed connection weight matrix, respectively, $\Delta A_{1}(t), \Delta B_{1}(t)$ are the time-varying parametric uncertainties, $J$ is the constant input vector, $f(x(\cdot))=\left(f_{1}\left(x_{1}(\cdot)\right), f_{2}\left(x_{2}(\cdot)\right), \ldots, f_{n}\left(x_{n}(\cdot)\right)\right)^{T}$ is the nonlinear neuron activation function which describes the behavior in which the neurons respond to each other, and the time delay $\tau(t)$ is bounded and continuously differentiable function; that is, there exist two positive constants $\tau$ and $\mu$, such that $0 \leq \tau(t) \leq \tau$ and $0 \leq \dot{\tau}(t) \leq \mu<1$.

For drive system (1), we construct the following response system with nonidentical parametric uncertainties and stochastic perturbation:

$$
\begin{aligned}
d y(t)=[ & -C y(t)+\left(A+\Delta A_{2}(t)\right) f(y(t)) \\
& \left.+\left(B+\Delta B_{2}(t)\right) f(y(t-\tau(t)))+u(t)+J\right] d t \\
+ & \sigma(t, y(t)-x(t), y(t-\tau(t)) \\
& -x(t-\tau(t))) d \omega(t),
\end{aligned}
$$

where $y(t)=\left(y_{1}(t), y_{2}(t), \ldots, y_{n}(t)\right)^{T}$ is the neuron state vector of the response system; $\omega(t)=\left(\omega_{1}(t), \ldots, \omega_{n}(t)\right)^{T}$ is an $n$ dimensional Brown motion defined on a complete probability space $(\Omega, \mathscr{F}, \mathscr{P})$ with a natural filtration $\left\{\mathscr{F}_{t}\right\}_{t \geq 0}$ (i.e., $\mathscr{F}_{t}=$ $\sigma\{\omega(s): 0 \leq s \leq t\})$, which satisfies $E\{d \omega(t)\}=0$ and $E\left\{[d \omega(t)]^{2}\right\}=d t ; \sigma: \mathbb{R}^{+} \times \mathbb{R}^{n} \times \mathbb{R}^{n} \rightarrow \mathbb{R}^{n \times n}$ is the noise intensity matrix; $u(t)$ is the robust adaptive controller which will be designed to compensate the nonidentical parametric uncertainties.

Let $e(t)=y(t)-x(t)$ be the synchronization error; then we can obtain the error system between systems (1) and (2) as follows:

$$
\begin{aligned}
d e(t)=[ & -C e(t)+\left(A+\Delta A_{2}(t)\right) h(e(t)) \\
& +\left(B+\Delta B_{2}(t)\right) h(e(t-\tau(t))) \\
& +\left(\Delta A_{2}(t)-\Delta A_{1}(t)\right) f(x(t)) \\
& \left.+\left(\Delta B_{2}(t)-\Delta B_{1}(t)\right) f(x(t-\tau(t)))+u(t)\right] d t \\
& +\sigma(t, e(t), e(t-\tau(t))) d \omega(t),
\end{aligned}
$$

where $h(e(\cdot))=f(e(\cdot)+x(\cdot))-f(x(\cdot))$. 
Throughout this paper, we assume that

(H1) [32] each neuron activation function $f_{i}: \mathbb{R} \rightarrow \mathbb{R}$ is continuous with $f_{i}(0)=0(i=1,2, \ldots, n)$, and there exist positive scalars $l_{i}$, such that

$$
\left|f_{i}(a)-f_{i}(b)\right| \leq l_{i}|a-b|
$$

for any $a, b \in \mathbb{R}, a \neq b$, and $L=\operatorname{diag}\left(l_{1}, l_{2}, \ldots, l_{n}\right)$;

(H2) the noise matrix $\sigma(\cdot)$ is local Lipschitz continuous and satisfies the linear growth condition as well, and $\sigma(t, 0,0)=0$. Moreover, there exist two real positive $n \times n$ matrices $Q_{1}, Q_{2}$, such that

$\operatorname{trace}\left[\sigma^{T}(t, u, v) \sigma(t, u, v)\right] \leq u^{T} Q_{1} u+v^{T} Q_{2} v$,

for all $u, v \in \mathbb{R}^{n}$;

(H3) the parametric uncertainties $\Delta A_{j}(t), \Delta B_{j}(t), j=1,2$ are in terms of

$$
\Delta A_{j}(t)=E_{j} F_{j}(t) H_{j}, \quad \Delta B_{j}(t)=E_{j}^{\prime} F_{j}^{\prime}(t) H_{j}^{\prime},
$$

where $E_{j}, E_{j}^{\prime}, H_{j}$, and $H_{j}^{\prime}$ are known real constant matrices with appropriate dimensions and $F_{j}(t)$ and $F_{j}^{\prime}(t)$ are the uncertain matrices which are Lebesque measurable in $t$ and satisfy $F_{j}^{T}(t) F_{j}(t) \leq I$ and $F_{j}^{\prime T}(t) F_{j}^{\prime}(t) \leq I$;

(H4) there exist unknown constants $\bar{\alpha}>0, \bar{\beta}>0$, such that

$$
\left\|\Delta A_{2}(t)-\Delta A_{1}(t)\right\| \leq \bar{\alpha}, \quad\left\|\Delta B_{2}(t)-\Delta B_{1}(t)\right\| \leq \bar{\beta} .
$$

Remark 1. Considering Hypothesis (H1) and the boundedness of the chaotic signals, one can get that there exist positive constants $l=\max _{1 \leq i \leq n} l_{i}$ and $\|x\| \leq \chi$, such that

$$
\|f(x(t))\| \leq l \chi, \quad\|f(x(t-\tau(t)))\| \leq l \chi .
$$

Remark 2. Let $e(t ; \xi)$ denote the state trajectory from the initial data $e(\theta)=\xi(\theta)$ on $-\tau \leq \theta \leq 0$ in $C_{\mathscr{F}_{0}}^{2}\left([-\tau, 0] ; \mathbb{R}^{n}\right)$. Although $\sigma(t, 0,0)=0$ and $f_{i}(0)=0$, system (3) would not admit a unique solution or trivial solution $e(t ; 0)=0$. Thus, Hypothesis (H2) in [31] is not satisfied. Therefore, the LaSalle invariance principle for stochastic differential delay equation [31, Corollary 3.1] cannot be applied for the stability analysis of the error system (3) and other stochastic delayed systems without a trivial solution $e(t ; 0)=0$, which implies that the methods proposed in $[26,33,34]$ are debatable. In order to release the restriction of the nonexistence of a trivial solution, we turn to utilize Gronwally's inequality to ensure the rigorousness of mathematical proof.

The following definition and lemmas are useful for future derivations.
Definition 3. If the function $V \in C^{1,2}\left(\mathbb{R}^{+} \times \mathbb{R}^{n} ; \mathbb{R}^{+}\right)$, then an operator $\mathfrak{Q} V$ from $\mathbb{R}^{+} \times \mathbb{R}^{n}$ along the trajectory of the error system (3) is defined as

$$
\begin{aligned}
& \mathfrak{Q V}(t, e(t)) \\
& \begin{aligned}
& V_{t}(t, e(t))+V_{e}(t, e(t)) \\
& \times[-C e(t)+\left(A+\Delta A_{2}(t)\right) h(e(t)) \\
&+\left(B+\Delta B_{2}(t)\right) h(e(t-\tau(t))) \\
&+\left(\Delta A_{2}(t)-\Delta A_{1}(t)\right) f(x(t)) \\
&\left.+\left(\Delta B_{2}(t)-\Delta B_{1}(t)\right) f(x(t-\tau(t)))+u(t)\right] \\
&+\frac{1}{2} \operatorname{trace}\left[\sigma ^ { T } \left(t, e(t), e(t-\tau(t)) V_{e e}\right.\right. \\
& \times(t, e(\mathrm{t})) \sigma^{T}(t, e(t), e(t-\tau(t))],
\end{aligned}
\end{aligned}
$$

where

$$
\begin{gathered}
V_{t}(t, e(t))=\frac{\partial V(t, e(t))}{\partial t}, \\
V_{e}(t, e(t))=\left(\frac{\partial V(t, e(t))}{\partial e_{1}}, \ldots, \frac{\partial V(t, e(t))}{\partial e_{n}}\right), \\
V_{e e}(t, e(t))=\left(\frac{\partial^{2} V(t, e(t))}{\partial e_{i} \partial e_{j}}\right)_{n \times n} .
\end{gathered}
$$

Definition 4. Systems (1) and (2) are said to be exponentially synchronized in mean square if there exist constants $\lambda>$ 0 and $\mathscr{M}>0$, such that $E\|e(t)\|^{2} \leq \mathscr{M} \exp (-\lambda t)$ and $t>0$, where $\lambda$ is called the decay rate of exponential synchronization.

Lemma 5 (Gronwally's inequality (see [35])). Let $T>0$ and $u(\cdot)$ be a Borel measurable bounded nonnegative function on $[0, T]$. If

$$
u(t) \leq c+v \int_{0}^{t} u(s) d s, \quad \forall 0 \leq t \leq T
$$

for some constants $c$, $v$, then

$$
u(t) \leq c \exp (v t), \quad \forall 0 \leq t \leq T .
$$

Lemma 6 (see [36]). If $X$ and $Y$ are real matrices with appropriate dimensions, then there exists a number $\varepsilon>0$, such that

$$
X^{T} Y+Y^{T} X \leq \varepsilon X^{T} X+\frac{1}{\varepsilon} Y^{T} Y .
$$

\section{Main Results}

In this section, the robust adaptive exponential synchronization in mean square for the drive system (1) and response system (2) is studied under Hypotheses $(H 1)-(H 4)$. The robust adaptive feedback controller is designed as

$$
\begin{aligned}
u(t)= & -K e(t)-l \chi\left\|e^{T} P\right\|^{-1} P e(t) \hat{\alpha} \\
& -l \chi\left\|e^{T} P\right\|^{-1} P e(t) \widehat{\beta},
\end{aligned}
$$


where $K$ is the feedback gain matrix to be scheduled; $\widehat{\alpha}$ and $\widehat{\beta}$ are the estimation of the bounds $\bar{\alpha}$ and $\bar{\beta}$, respectively. The update laws $\widehat{\alpha}$ and $\widehat{\beta}$ are designed as

$$
\dot{\hat{\alpha}}=k_{\alpha} l \chi\left\|e^{T} P\right\|, \quad \dot{\hat{\beta}}=k_{\beta} l \chi\left\|e^{T} P\right\|,
$$

with $k_{\alpha}, k_{\beta}>0$.

Theorem 7. Assume that Hypotheses (H1)-(H4) hold. If there exist an $n \times n$ feedback gain matrix $K$, two $n \times n$ symmetric matrices $P>0$ and $R>0$, and four positive constants $\rho, \theta_{1}$, $\theta_{2}$, and $\kappa$, such that the following conditions hold:

(C1) $P \leq \rho I$;

(C2)

$$
\left[\begin{array}{ccccc}
\Omega_{11} & P A & P E_{2} & P B & P E_{2}^{\prime} \\
\star & -\frac{1}{\theta_{1}} I & 0 & 0 & 0 \\
\star & \star & -\frac{1}{\left(\theta_{1}\left\|H_{2}\right\|^{2}\right)} & 0 & 0 \\
\star & \star & \star & -\frac{1}{\theta_{2}} I & 0 \\
\star & \star & \star & \star & -\frac{1}{\left(\theta_{2}\left\|H_{2}^{\prime}\right\|^{2}\right)}
\end{array}\right] \leq 0,
$$

where

$$
\Omega_{11}=P(-K-C)+(-K-C)^{T} P+\frac{2}{\theta_{1}} L^{2}+R+\rho Q_{1}+\kappa I,
$$

then the drive system (1) and response system (2) can be exponentially synchronized in mean square.

Proof. Construct a Lyapunov functional in the form of

$$
\begin{aligned}
V(t, e(t))= & e^{T}(t) P e(t) \\
& +\int_{t-\tau(t)}^{t} e^{T}(s) \operatorname{Re}(s) d s+k_{\alpha}^{-1} \widetilde{\alpha}^{2}+k_{\beta}^{-1} \widetilde{\beta}^{2},
\end{aligned}
$$

where $\widetilde{\alpha}=\bar{\alpha}-\widehat{\alpha}, \widetilde{\beta}=\bar{\beta}-\widehat{\beta}$.

From Definition 3, we obtain the operator $\mathfrak{Q} V(t, e(t))$

$$
\begin{aligned}
& \mathfrak{Q} V(t, e(t)) \\
& =2 e^{T}(t) P\left(-C e(t)+\left(A+\Delta A_{2}(t)\right) h(e(t))\right. \\
& +\left(B+\Delta B_{2}(t)\right) h(e(t-\tau(t))) \\
& +\left(\Delta A_{2}(t)-\Delta A_{1}(t)\right) f(x(t)) \\
& \left.+\left(\Delta B_{2}(t)-\Delta B_{1}(t)\right) f(x(t-\tau(t)))+u(t)\right) \\
& +\operatorname{trace}\left[\sigma^{T}(t, e(t), e(t-\tau(t)))\right. \\
& \times P \sigma(t, e(t), e(t-\tau(t)))]
\end{aligned}
$$

$$
\begin{aligned}
& +e^{T}(t) \operatorname{Re}(t)-(1-\dot{\tau}(t)) \\
& \times e^{T}(t-\tau(t)) \operatorname{Re}(t-\tau(t)) \\
& -\left(2 k_{\alpha}^{-1} \tilde{\alpha} \dot{\hat{\alpha}}+2 k_{\beta}^{-1} \tilde{\beta} \dot{\hat{\beta}}\right) \\
& =e^{T}(t)\left(-P C-C^{T} P+R\right) e(t) \\
& +2 e^{T}(t) P\left(A+\Delta A_{2}(t)\right) h(e(t)) \\
& +2 e^{T}(t) P\left(B+\Delta B_{2}(t)\right) h(e(t-\tau(t))) \\
& +2 e^{T}(t) P\left(\Delta A_{2}(t)-\Delta A_{1}(t)\right) f(x(t)) \\
& +2 e^{T}(t) P\left(\Delta B_{2}(t)-\Delta B_{1}(t)\right) \\
& \times f(x(t-\tau(t)))+2 e^{T}(t) P u(t) \\
& +\operatorname{trace}\left[\sigma^{T}(t, e(t), e(t-\tau(t)))\right. \\
& \quad \times P \sigma(t, e(t), e(t-\tau(t)))] \\
& -(1-\dot{\tau}(t)) e^{T}(t-\tau(t)) \operatorname{Re}(t-\tau(t)) \\
& -\left(2 k_{\alpha}^{-1} \tilde{\alpha} \dot{\hat{\alpha}}+2 k_{\beta}^{-1} \tilde{\beta} \dot{\hat{\beta}}\right) .
\end{aligned}
$$

It is clear from Lemma 6 that

$$
\begin{aligned}
& 2 e^{T}(t) P\left(A+\Delta A_{2}(t)\right) h(e(t)) \\
& \leq \theta_{1} e^{T}(t) P A A^{T} P e(t) \\
&+\frac{1}{\theta_{1}} h^{T}(e(t)) h(e(t)) \\
&+\theta_{1} e^{T}(t) P E_{2} F_{2}(t) H_{2} H_{2}^{T} F_{2}^{T}(t) E_{2}^{T} P e(t) \\
&+\frac{1}{\theta_{1}} h^{T}(e(t)) h(e(t)) \\
& \leq \theta_{1} e^{T}(t) P A A^{T} P e(t)+\frac{2}{\theta_{1}} e^{T}(t) L^{2} e(t) \\
&+\theta_{1}\left\|H_{2}\right\|^{2} e^{T}(t) P E_{2} E_{2}^{T} P e(t) \\
&= e^{T}(t)\left[\theta_{1}\left(P A A^{T} P+\left\|H_{2}\right\|^{2} P E_{2} E_{2}^{T} P\right)\right. \\
&\left.+\frac{2}{\theta_{1}} L^{2}\right] e(t), \\
& 2 e^{T}(t) P\left(B+\Delta B_{2}(t)\right) h(e(t-\tau(t))) \\
& \leq \theta_{2} e^{T}(t) P B B^{T} P e(t)
\end{aligned}
$$




$$
\begin{aligned}
& +\frac{1}{\theta_{2}} h^{T}(e(t-\tau(t))) h(e(t-\tau(t))) \\
& +\theta_{2} e^{T}(t) P E_{2}^{\prime} F_{2}^{\prime}(t) H_{2}^{\prime} H_{2}^{\prime T} F_{2}^{\prime T}(t) E_{2}^{\prime T} P e(t) \\
& +\frac{1}{\theta_{2}} h^{T}(e(t-\tau(t))) h(e(t-\tau(t))) \\
\leq & \theta_{2} e^{T}(t) P B B^{T} P e(t) \\
& +\frac{2}{\theta_{2}} e^{T}(t-\tau(t)) L^{2} e(t-\tau(t)) \\
& +\theta_{2}\left\|H_{2}^{\prime}\right\|^{2} e^{T}(t) P E_{2}^{\prime} E_{2}^{\prime T} P e(t) \\
= & e^{T}(t)\left[\theta_{2}\left(P B B^{T} P+\left\|H_{2}^{\prime}\right\|^{2} P E_{2}^{\prime} E_{2}^{\prime T} P\right)\right] e(t) \\
& +\frac{2}{\theta_{2}} e^{T}(t-\tau(t)) L^{2} e(t-\tau(t)), \\
2 e^{T}( & t) P\left(\Delta A_{2}(t)-\Delta A_{1}(t)\right) f(x(t)) \\
& +2 e^{T}(t) P\left(\Delta B_{2}(t)-\Delta B_{1}(t)\right) f(x(t-\tau(t))) \\
\leq & 2\left\|e^{T} P\right\| \bar{\alpha} l \chi+2\left\|e^{T} P\right\| \bar{\beta} l \chi \\
= & 2\left\|e^{T} P\right\| l \chi(\bar{\alpha}+\bar{\beta}) .
\end{aligned}
$$

From Hypothesis (H3), we have

$$
\begin{aligned}
\operatorname{trace}\left[\sigma^{T} P \sigma\right] & \leq \lambda_{\max }(P) \operatorname{trace}\left[\sigma^{T} \sigma\right] \\
& \leq \rho\left(e^{T}(t) Q_{1} e(t)\right. \\
& \left.+e^{T}(t-\tau(t)) Q_{2} e(t-\tau(t))\right) .
\end{aligned}
$$

Substituting (14) and (15), (20) and (21) to (19), we obtain

$$
\begin{aligned}
\mathcal{L V}( & t, e(t)) \\
\leq & e^{T}(t) \Omega_{1} e(t) \\
& +e^{T}(t-\tau(t)) \Omega_{2} e(t-\tau(t)) \\
& +2\left\|e^{T} P\right\| l \chi(\bar{\alpha}+\bar{\beta}) \\
& \quad-2 e^{T}(t) P\left(l \chi\left\|e^{T} P\right\|^{-1} P e(t) \widehat{\alpha}\right. \\
& \left.\quad+l \chi\left\|^{T} P\right\|^{-1} P e(t) \widehat{\beta}\right) \\
& -2 k_{\alpha}^{-1} \widetilde{\alpha} k_{\alpha} l \chi\left\|e^{T} P\right\|-2 k_{\beta}^{-1} \widetilde{\beta} k_{\beta} l \chi\left\|e^{T} P\right\| \\
= & e^{T}(t) \Omega_{1} e(t)+e^{T}(t-\tau(t)) \Omega_{2} e(t-\tau(t)) \\
& +2\left\|e^{T} P\right\| l \chi(\bar{\alpha}+\bar{\beta}-\widehat{\alpha}-\widehat{\beta})-2\left\|e^{T} P\right\| l \chi(\widetilde{\alpha}+\widetilde{\beta}) \\
= & e^{T}(t) \Omega_{1} e(t)+e^{T}(t-\tau(t)) \Omega_{2} e(t-\tau(t)) \\
= & e^{T}(t)\left(\Omega_{1}+\kappa I\right) e(t)+e^{T}(t-\tau(t)) \Omega_{2} e(t-\tau(t)) \\
& -\kappa e^{T}(t) e(t) \\
= & e^{T}(t) \Omega_{1}^{\prime} e(t)+e^{T}(t-\tau(t)) \Omega_{2} e(t-\tau(t)) \\
& -\kappa e^{T}(t) e(t),
\end{aligned}
$$

where

$$
\begin{aligned}
\Omega_{1}= & P(-K-C)+(-K-C)^{T} P \\
& +R+\rho Q_{1}+\frac{2}{\theta_{1}} L^{2} \\
& +\theta_{1}\left(P A A^{T} P+\left\|H_{2}\right\|^{2} P E_{2} E_{2}^{T} P\right) \\
& +\theta_{2}\left(P B B^{T} P+\left\|H_{2}^{\prime}\right\|^{2} P E_{2}^{\prime} E_{2}^{\prime T} P\right), \\
\Omega_{1}^{\prime}= & \Omega_{1}+\kappa I, \\
\Omega_{2}= & \frac{2}{\theta_{2}} L^{2}+\rho Q_{2}-(1-\mu) R .
\end{aligned}
$$

Considering condition (C2) of Theorem 7 and Schur complement [37], we get

$$
\mathfrak{Q} V(t, e(t)) \leq-\kappa e^{T}(t) e(t) .
$$

It follows from Itô's formula that

$$
\begin{aligned}
E V & (t, e(t))-E V(0, e(0)) \\
& =\int_{0}^{t} E \mathfrak{Q} V \leq-\kappa \int_{0}^{t} E\left(e(s)^{T} e(s)\right) d s,
\end{aligned}
$$

$t>0$,

which means that

$\lambda_{\min }(P) E\|e(t)\|^{2} \leq E V(t, e(t))$

$$
\leq E V(0, e(0))-\kappa \int_{0}^{t} E\|e(s)\|^{2} d s .
$$

By using Lemma 5, we have

$$
E\|e(t)\|^{2} \leq c \exp (v t),
$$

where $c=E V(0, e(0)) / \lambda_{\text {min }}(P), v=-\kappa / \lambda_{\text {min }}(P)$.

On the other hand, we denote

$$
\begin{aligned}
E V(0, e(0))= & E e^{T}(0) \operatorname{Pe}(0) \\
& +\int_{-\tau(0)}^{0} E e^{T}(s) \operatorname{Re}(s) \\
& +k_{\alpha}^{-1} \widetilde{\alpha}^{2}(0)+k_{\beta}^{-1} \widetilde{\beta}^{2}(0) \\
\leq & \lambda_{\max }(P) E \sup _{-\tau \leq \theta \leq 0}\|\xi(\theta)\|^{2} \\
& +\tau \lambda_{\max }(R) E \sup _{-\tau \leq \theta \leq 0}\|\xi(\theta)\|^{2} \\
& +k_{\alpha}^{-1} \widetilde{\alpha}^{2}(0)+k_{\beta}^{-1} \widetilde{\beta}^{2}(0) \\
= & \left(\lambda_{\max }(P)+\tau \lambda_{\max }(R)\right) E \sup _{-\tau \leq \theta \leq 0}\|\xi(\theta)\|^{2} \\
& +k_{\alpha}^{-1} \widetilde{\alpha}^{2}(0)+k_{\beta}^{-1} \widetilde{\beta}^{2}(0) .
\end{aligned}
$$


Combining (27) and (28), we finally obtain

$$
E\|e(t)\|^{2} \leq \mathscr{M} \exp \left(\frac{-\kappa}{\lambda_{\min }(P)} t\right),
$$

where

$$
\begin{aligned}
\mathscr{M}=c=(( & \left.\lambda_{\max }(P)+\tau \lambda_{\max }(R)\right) E \sup _{-\tau \leq \theta \leq 0}\|\xi(\theta)\|^{2} \\
& \left.+k_{\alpha}^{-1} \widetilde{\alpha}^{2}(0)+k_{\beta}^{-1} \widetilde{\beta}^{2}(0)\right) \times\left(\lambda_{\min }(P)\right)^{-1} .
\end{aligned}
$$

Therefore, by Definition 4 we see that the drive system (1) and response system (2) can be exponentially synchronized in mean square with a decay rate $\lambda=\kappa / \lambda_{\text {min }}(P)$. The proof of Theorem 7 is completed.

Let $Y=P K$; the feedback gain matrix $K$ can be calculated by $K=P^{-1} Y$; then, the following Theorem holds.

Theorem 8. Assume that Hypotheses (H1)-(H4) hold. If there exist an $n \times n$ matrix $Y$, two $n \times n$ symmetric matrices $P>0$ and $R>0$, and four positive constants $\rho, \theta_{1}, \theta_{2}$, and $\kappa$, such that the following conditions hold

(C1) $P \leq \rho I$;

$\left(C 2^{\prime}\right)$

$$
\begin{aligned}
& {\left[\begin{array}{ccccc}
\Omega_{11}^{\prime} & P A & P E_{2} & P B & P E_{2}^{\prime} \\
\star & -\frac{1}{\theta_{1}} I & 0 & 0 & 0 \\
\star & \star & -\frac{1}{\left(\theta_{1}\left\|H_{2}\right\|^{2}\right)} & 0 & 0 \\
\star & \star & \star & -\frac{1}{\theta_{2}} I & 0 \\
\star & \star & \star & \star & -\frac{1}{\left(\theta_{2}\left\|H_{2}^{\prime}\right\|^{2}\right)}
\end{array}\right] \leq 0,} \\
& \frac{2}{\theta_{2}} L^{2}+\rho Q_{2}-(1-\mu) R \leq 0,
\end{aligned}
$$

where

$$
\Omega_{11}^{\prime}=-P C-Y-C^{T} P-Y^{T}+\frac{2}{\theta_{1}} L^{2}+R+\rho Q_{1}+\kappa I,
$$

then the drive system (1) and response system (2) can be exponentially synchronized in mean square.

Substituting $K=P^{-1} Y$ to Theorem 7 , the above result is easy to be obtained. So the proof of Theorem 8 is omitted.

Remark 9. Our method does not need to construct a complex Lyapunov-Krasovskii function which contains quadratic integral terms and triple integral terms. Thus, the amount of calculation is greatly reduced. Furthermore, we do not require that the matrix $P>0$ is diagonal (see $[8,9$, 30]) just only being symmetric. This partly shows the less conservativeness of our control strategy. If the drive system (1) and response system (2) have no nonidentical parametric uncertainties, the corresponding synchronization problems have been addressed in $[8,9,29,30]$. Therefore, our results are more general than those given in $[8,9,29,30]$.

By constructing another Lyapunov functional $V^{\prime}(t, e(t))=e^{T}(t) P e(t)+\int_{t-\tau(t)}^{t} e^{T}(s) \operatorname{Re}(s) d s$, we can derive the following corollaries by Theorem 7 and Theorem 8, respectively.

Corollary 10. Assume that Hypotheses (H1)-(H4) hold, and the feedback controller is designed as $u(t)=-K(y(t)-x(t))$. If there exist an $n \times n$ feedback gain matrix $K$, two $n \times n$ symmetric matrices $P>0$ and $R>0$, and four positive constants $\rho, \theta_{1}$, $\theta_{2}$, and $\kappa$, such that the following conditions hold

(i) $P \leq \rho I$;

(ii)

$$
\begin{aligned}
& {\left[\begin{array}{ccc}
\Xi_{11} & P A & P B \\
\star & -\frac{1}{\theta_{1}} I & 0 \\
\star & \star & -\frac{1}{\theta_{2}} I
\end{array}\right] \leq 0,} \\
& \frac{2}{\theta_{2}} L^{2}+\rho Q_{2}-(1-\mu) R \leq 0,
\end{aligned}
$$

where

$$
\begin{aligned}
\Xi_{11}= & P(-K-C)+(-K-C)^{T} P \\
& +\frac{2}{\theta_{1}} L^{2}+R+\rho Q_{1}+\kappa I,
\end{aligned}
$$

then the drive system (1) and response system (2) can be exponentially synchronized in mean square.

Corollary 11. Assume that Hypotheses (H1)-(H4) hold, and the feedback controller is designed as $u(t)=-K(y(t)-x(t))$. If there exist an $n \times n$ matrix $Y$, two $n \times n$ symmetric matrices $P>0$ and $R>0$, and four positive constants $\rho, \theta_{1}, \theta_{2}$, and $\kappa>0$, such that the following conditions hold

(i) $P \leq \rho I$;

$\left(i i^{\prime}\right)$

$$
\begin{aligned}
& {\left[\begin{array}{ccc}
\Xi_{11}^{\prime} & P A & P B \\
\star & -\frac{1}{\theta_{1}} I & 0 \\
\star & \star & -\frac{1}{\theta_{2}} I
\end{array}\right] \leq 0,} \\
& \frac{2}{\theta_{2}} L^{2}+\rho Q_{2}-(1-\mu) R \leq 0,
\end{aligned}
$$


where

$$
\begin{gathered}
\Xi_{11}^{\prime}=-P C-Y-C^{T} P-Y^{T}+\frac{2}{\theta_{1}} L^{2}+R+\rho Q_{1}+\kappa I, \\
Y=P K
\end{gathered}
$$

then the drive system (1) and response system (2) can be exponentially synchronized in mean square.

The proof of Corollaries 10 and 11 is direct, so it is omitted.

Remark 12. Suppose response system (2) without noise perturbation; then, the synchronization issue of the drive system (1) and response system (2) converted to the synchronization of chaotic delayed neural networks with parametric uncertainties, which has been investigated in [11, 23]. In addition, the authors in [33] only studied the time invariance parametric uncertainties. Subsequently, our method has a wider application range than those results in [11, 23, 33].

\section{Numerical Results}

In this section, a numerical example and its simulations are presented to demonstrate the effectiveness of the proposed scheme in the previous sections. Throughout the simulations, we use the Euler-Maruyama method [38] with step $10^{-3}$.

Example 13. Consider two-dimensional chaotic delayed neural networks [39] as the drive system:

$$
\begin{aligned}
d x(t)=[- & C x(t)+\left(A+\Delta A_{1}(t)\right) f(x(t)) \\
& \left.+\left(B+\Delta B_{1}(t)\right) f(x(t-\tau(t)))+J\right] d t,
\end{aligned}
$$

where the initial condition $x(s)=(0.1,0.1)^{T}, s \in[-\tau, 0]$, $f(x)=0.5(|x+1|-|x-1|)$, and $\tau(t)=1, J=(0,0)^{T}$; then, from Hypothesis $(H 1)$ and Remark 1 , it is easy to verify that $L_{1}=$ $(1,1)^{T}, L_{2}=(0,0)^{T}$, and $l=1$. The parameter matrices $C, A$, and $B$ and the parametric uncertainties are given as follows:

$$
\begin{aligned}
& C=\left(\begin{array}{ll}
1 & 0 \\
0 & 1
\end{array}\right), \quad A=\left(\begin{array}{cc}
1+\frac{\pi}{4} & 20 \\
0.1 & 1+\frac{\pi}{4}
\end{array}\right), \\
& B=\left(\begin{array}{cc}
-\sqrt{2} \times \frac{1.3 \pi}{4} & 0.1 \\
0.1 & -\sqrt{2} \times \frac{1.3 \pi}{4}
\end{array}\right), \\
& \Delta A_{1}(t)=0.04 *\left(\begin{array}{cc}
\sin (t) & 0 \\
0 & \sin (t)
\end{array}\right) \\
& \Delta B_{1}(t)=0.04 *\left(\begin{array}{cc}
\cos (t) & 0 \\
0 & \cos (t)
\end{array}\right)
\end{aligned}
$$

that is,

$$
\begin{aligned}
& F_{1}(t)=\left(\begin{array}{cc}
\sin (t) & 0 \\
0 & \sin (t)
\end{array}\right), \\
& F_{1}^{\prime}(t)=\left(\begin{array}{cc}
\cos (t) & 0 \\
0 & \cos (t)
\end{array}\right), \\
& E_{1}=H_{1}=E_{1}^{\prime}=H_{1}^{\prime}=0.2 I .
\end{aligned}
$$

The corresponding response system is taken as

$$
\begin{aligned}
d y(t)=[- & C y(t)+\left(A+\Delta A_{2}(t)\right) f(y(t)) \\
+ & \left(B+\Delta B_{2}(t)\right) f(y(t-\tau(t))) \\
+u(t)+J] d t+\sigma(t, y(t)-x(t), y(t-\tau(t)) & -x(t-\tau(t))) d \omega(t),
\end{aligned}
$$

where the initial condition $y(s)=(0.2,-0.2)^{T}, s \in[-\tau, 0]$, the noise perturbation

$$
\begin{aligned}
& \sigma(t, e(t), e(t-\tau(t))) \\
&= \operatorname{diag}\left(\sqrt{0.005} e_{1}(t), \sqrt{0.025} e_{2}(t)\right) \\
&+\operatorname{diag}\left(\sqrt{0.25} e_{1}(t-\tau(t)), \sqrt{0.005} e_{2}(t-\tau(t))\right),
\end{aligned}
$$

and $\omega(t)$ is a 2-dimensional Brownian motion satisfying $E\{d \omega(t)\}=0$ and $E\left\{[d \omega(t)]^{2}\right\}=d t$; then, from Hypothesis $(H 2)$, it is easy to get $Q_{1}=\operatorname{diag}(0.01,0.05)$ and $Q_{2}=$ $\operatorname{diag}(0.5,0.01)$. And the parametric uncertainties are given as

$$
\begin{aligned}
& \Delta A_{2}(t)=-0.04 *\left(\begin{array}{cc}
\sin (t) & 0 \\
0 & \sin (t)
\end{array}\right), \\
& \Delta B_{2}(t)=-0.04 *\left(\begin{array}{cc}
\cos (t) & 0 \\
0 & \cos (t)
\end{array}\right) ;
\end{aligned}
$$

that is,

$$
\begin{aligned}
& F_{2}(t)=-\left(\begin{array}{cc}
\sin (t) & 0 \\
0 & \sin (t)
\end{array}\right) \\
& F_{2}^{\prime}(t)=-\left(\begin{array}{cc}
\cos (t) & 0 \\
0 & \cos (t)
\end{array}\right) \\
& E_{2}=H_{2}=E_{2}^{\prime}=H_{2}^{\prime}=0.2 I
\end{aligned}
$$

The robust adaptive feedback controller $u(t)$ satisfies (14). The update laws $\widehat{\alpha}$ and $\widehat{\beta}$ are designed as (15). 


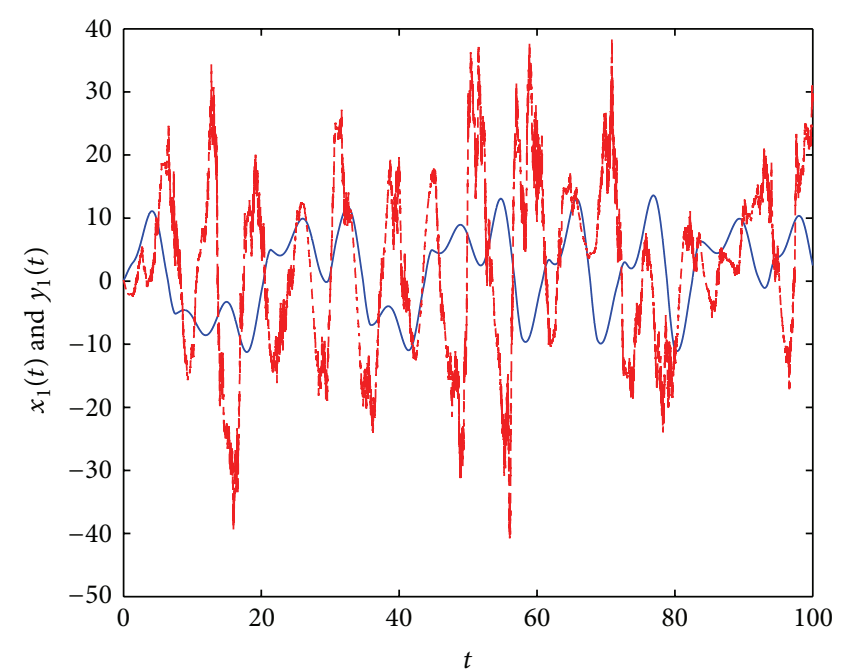

$\begin{aligned}- & x_{1}(t) \\ --- & y_{1}(t)\end{aligned}$

(a)

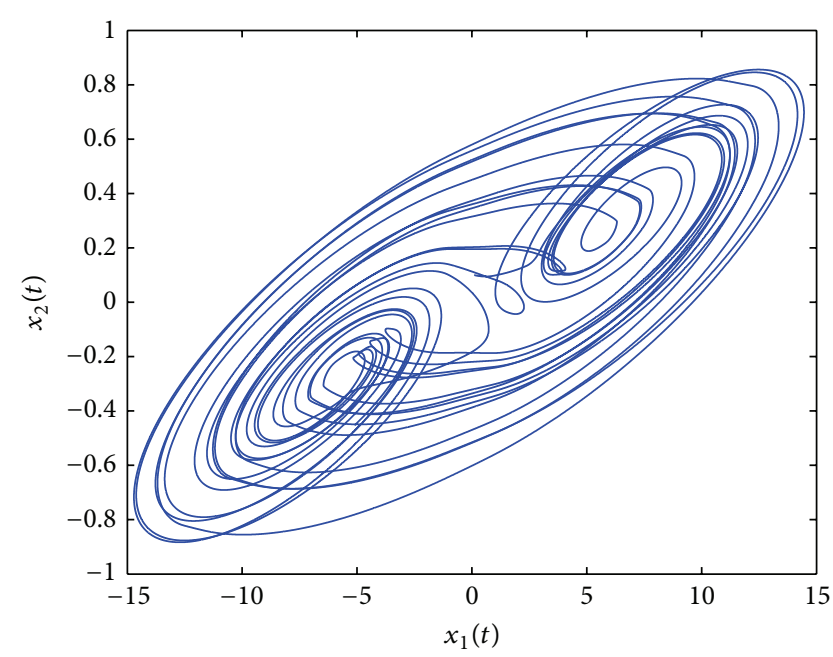

(c)

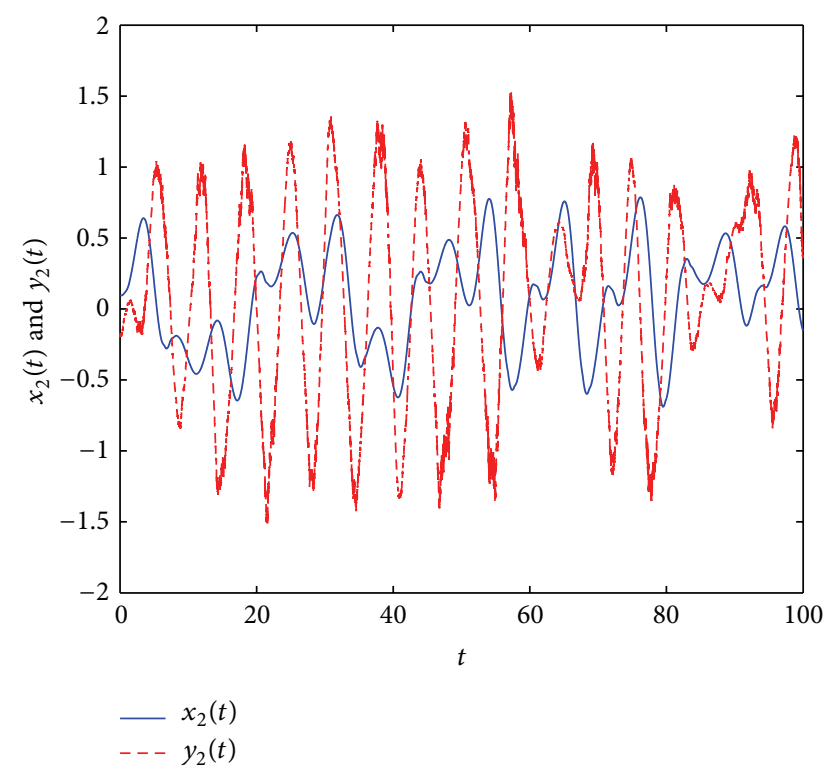

(b)

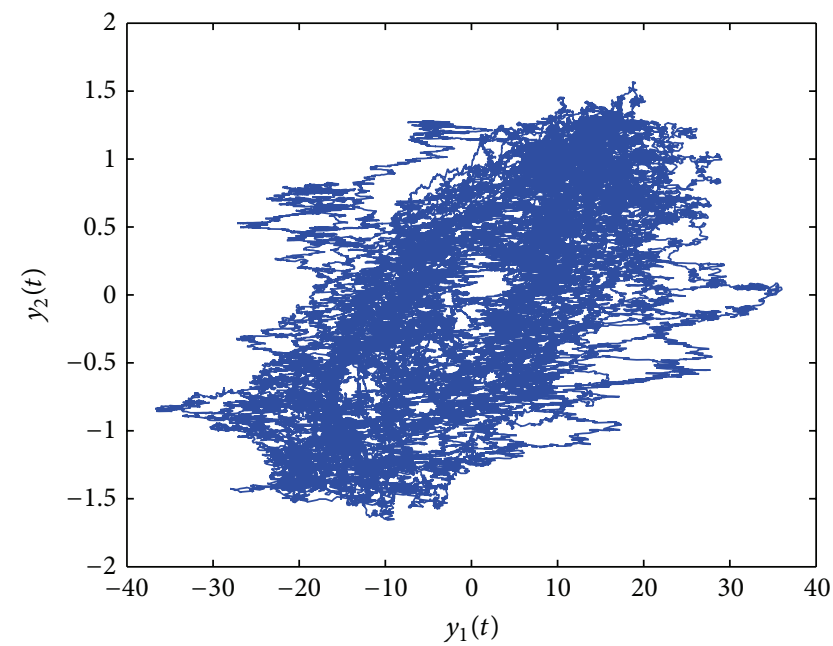

(d)

FIGURE 1: Time response of state variables and the phase plots of systems (37) and (40) without the robust adaptive feedback controller $u(t)$.

By using MATLAB LMI toolbox, we can obtain the following feasible solutions to LMIs in Theorem 8:

$$
\begin{gathered}
P=\left(\begin{array}{cc}
0.0040 & -0.0065 \\
-0.0065 & 0.0772
\end{array}\right), \quad Y=\left(\begin{array}{ll}
0.5206 & 0.0019 \\
0.0019 & 0.4607
\end{array}\right), \\
R=\left(\begin{array}{cc}
0.4307 & -0.0002 \\
-0.0002 & 0.3292
\end{array}\right), \\
\rho=0.2734, \quad \theta_{1}=6.1797, \\
\theta_{2}=9.4490, \quad \kappa=0.0842 .
\end{gathered}
$$

Thus, we can calculate the feedback gain matrix $K$ and the decay rate, which are given as follows:

$$
\begin{gathered}
K=P^{-1} Y=\left(\begin{array}{cc}
149.1244 & 11.6011 \\
12.5158 & 6.9422
\end{array}\right), \\
\lambda=\frac{\kappa}{\lambda_{\min }(P)}=24.2757 .
\end{gathered}
$$

We choose $\chi=16, k_{\alpha}=k_{\beta}=0.1, \widehat{\alpha}(0)=0.079$, and $\widehat{\beta}(0)=$ 0.079 . Figures $1(\mathrm{a})-1(\mathrm{~d})$ show the time response of state variables and the phase plots of systems (37) and (40) without the robust adaptive feedback controller $u(t)$ designed in (14). Figures 2(a)-2(d) depict the time response of state variables, the synchronization errors, and the phase plots of systems 


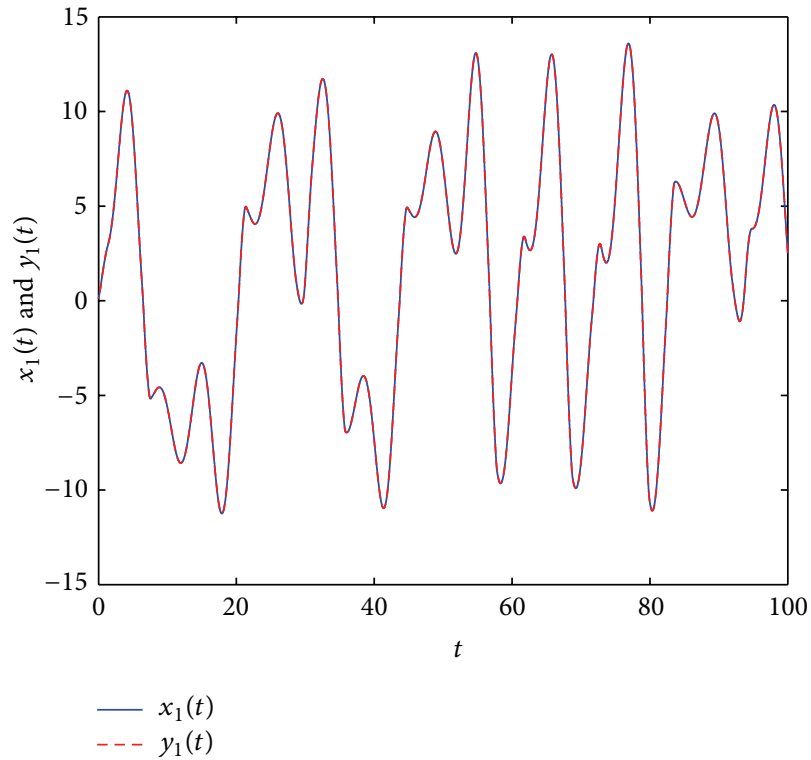

(a)

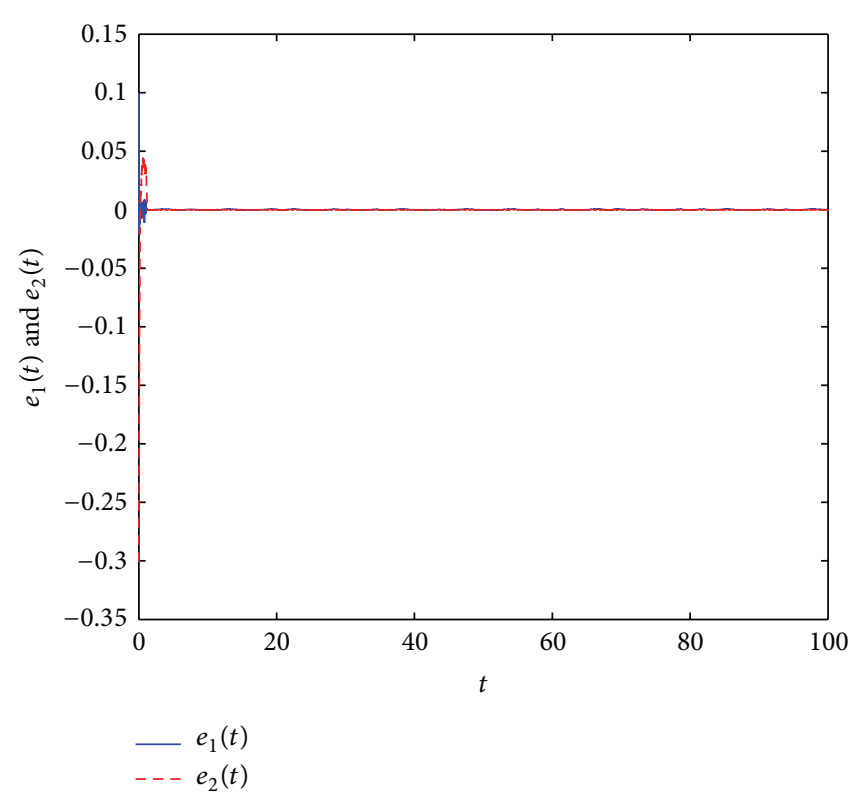

(c)

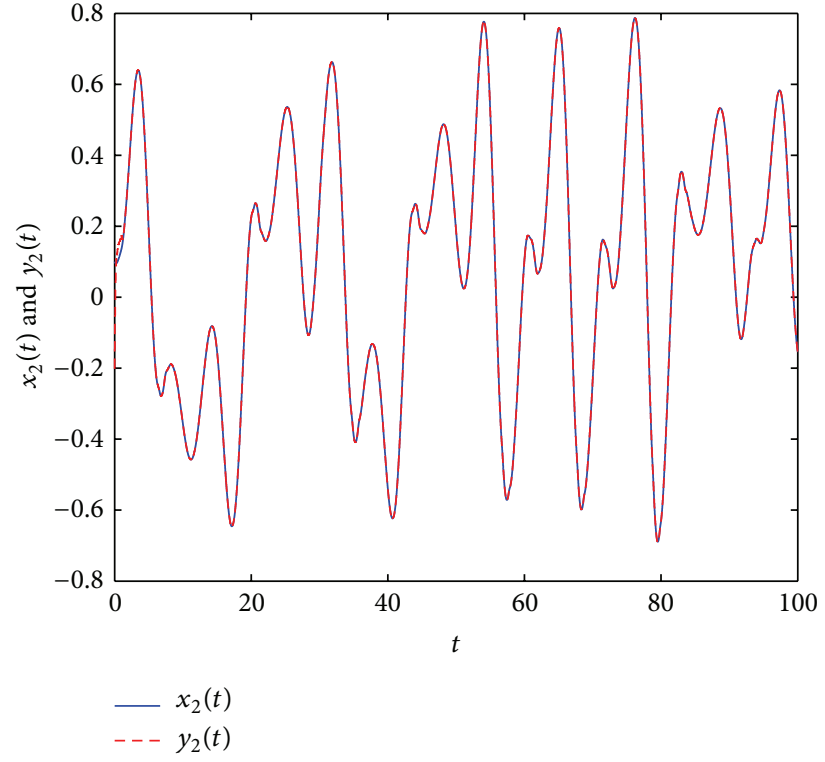

(b)

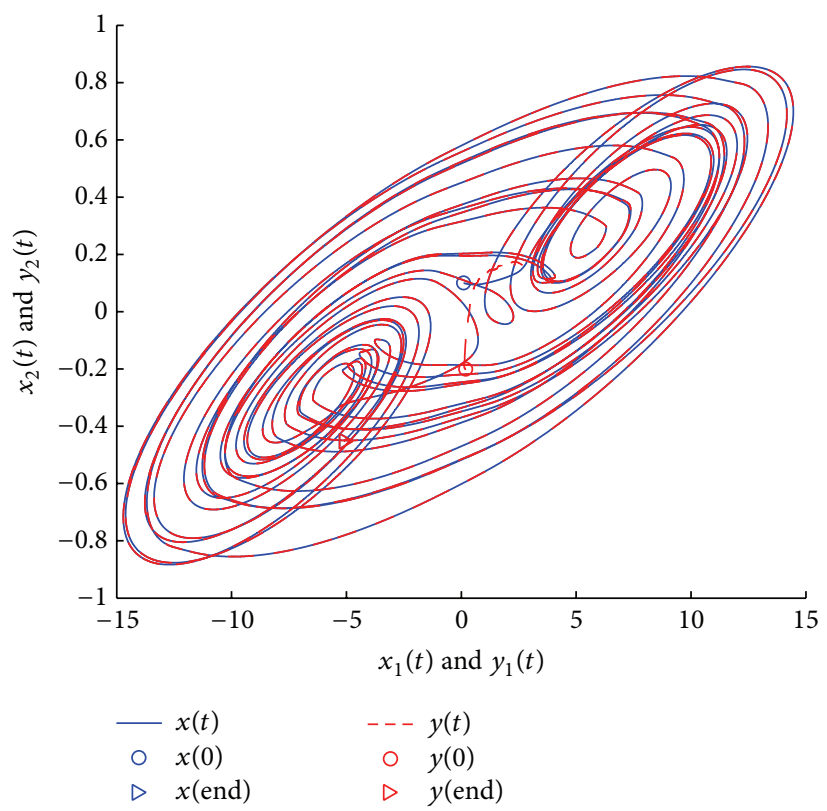

(d)

Figure 2: Time response of state variables, the synchronization errors, and the phase plots of systems (37) and (40) with the robust adaptive feedback controller $u(t)$.

(37) and (40) with the robust adaptive feedback controller $u(t)$ designed in (14), from which we can see that the drive system (37) and response system (40) can be exponentially synchronized in mean square. Figures $3(\mathrm{a})$ and $3(\mathrm{~b})$ display the estimated parameters $\widehat{\alpha}$ and $\widehat{\beta}$ converge asymptotically to some constants, which show the effectiveness of the proposed robust adaptive synchronization scheme.

Remark 14. The simulation results in Example 13 show that the effect of the nonidentical parametric uncertainties between the drive system (37) and response system (40) can be suppressed rapidly via the designed robust adaptive feedback controller, which means that the proposed strategy has strong robustness against the nonidentical parametric uncertainties. Meanwhile, we have a relatively large decay rate $\lambda$.

Remark 15. Synchronization is encountered in various fields of science, in engineering and in social behavior [40, 41]. In fact, synchronization problem of networks system belongs to the category of control, which is an important branch of 


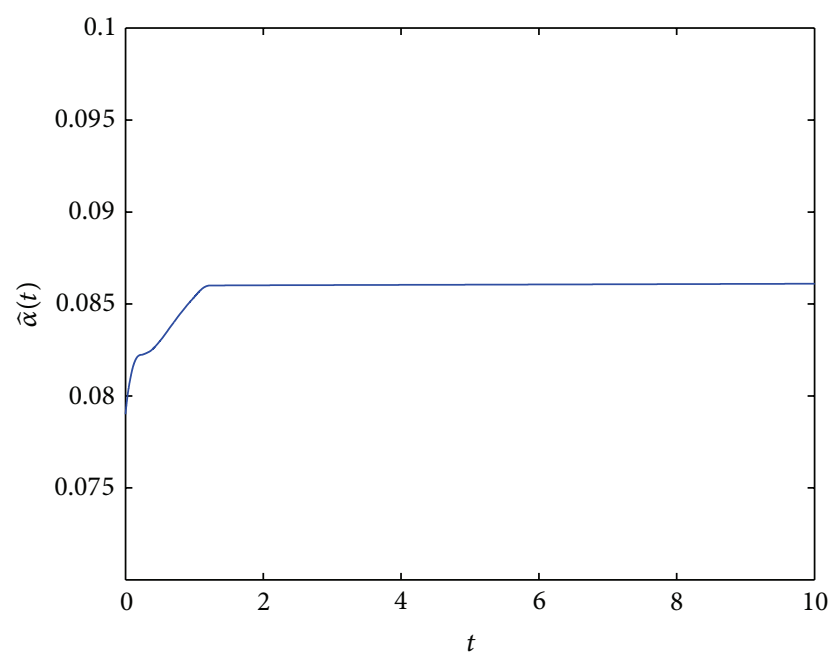

(a)

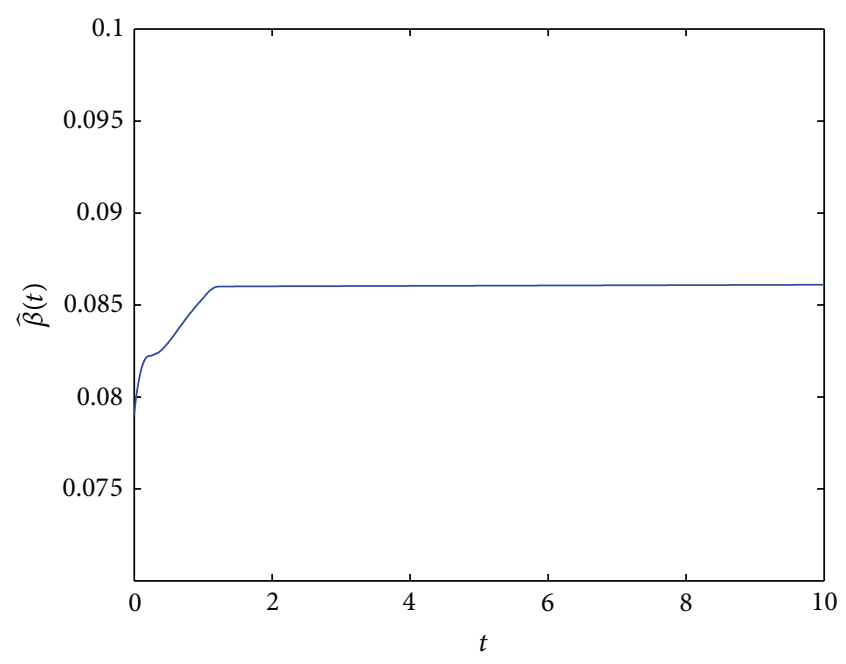

(b)

FIgURE 3: The curves of the estimated parameters $\widehat{\alpha}$ and $\widehat{\beta}$.

synthetic theory in model control field. The authors in [42] dealt with the synchronization problem of coupled switched neural networks with mode-dependent impulsive effects and time delays by using switching analysis techniques and a comparison principle. Zhang et al. in [43] have investigated the synchronization problem for a class of nonlinear delayed dynamical networks with heterogeneous impulsive effects. The distributed synchronization problem in networks of agent systems with controllers and nonlinearities subject to Bernoulli switchings has been studied in [44], in which the advantage of distributed adaptive controllers over conventional adaptive controllers has also been validated. The abovementioned literature is interesting synthesis problems of networks and will become our future investigative directions.

\section{Conclusion}

In this paper, we have studied robust exponential adaptive synchronization of stochastic perturbed chaotic delayed neural networks with nonidentical parametric uncertainties. A robust adaptive feedback controller has been designed to equalize the effect of the nonidentical parametric uncertainties. A numerical example has also been exploited to depict the usefulness of the obtained results. The nonidentical parametric uncertainties, which have been taken into account, exhibited the main advantage of the proposed scheme. The simulation results confirmed that our method has a high robustness property against parameter uncertainties mismatch.

\section{Conflict of Interests}

The authors declare that there is no conflict of interests regarding the publication of this paper.

\section{Acknowledgments}

The authors would like to thank the editor and the anonymous reviewers for their careful comments and suggestions to improve the quality of the paper. This work is jointly supported by Opening Fund of Geomathematics Key Laboratory of Sichuan Province under Grant sc-sxdz2011010 and the scientific research Fund of Sichuan University of Science and Engineering under Grant 2011PY08.

\section{References}

[1] C.-J. Cheng and C.-B. Cheng, "An asymmetric image cryptosystem based on the adaptive synchronization of an uncertain unified chaotic system and a cellular neural network," Communications in Nonlinear Science and Numerical Simulation, vol. 18, no. 10, pp. 2825-2837, 2013.

[2] V. Perez-Munuzuri, V. Perez-Villar, and L. O. Chua, "Autowaves for image processing on a two-dimensional CNN array of excitable nonlinear circuits: flat and wrinkled labyrinths," IEEE Transactions on Circuits and Systems I: Fundamental Theory and Applications, vol. 40, no. 3, pp. 174-181, 1993.

[3] J. J. Fox, C. Jayaprakash, D. Wang, and S. R. Campbell, "Synchronization in relaxation oscillator networks with conduction delays," Neural Computation, vol. 13, no. 5, pp. 1003-1021, 2001.

[4] V. Milanovic and M. E. Za-ghloul, "Synchronization of chaotic neural networks and applications to communications," International Journal of Bifurcation and Chaos, vol. 6, no. 12, pp. 25712585, 1996.

[5] T. Yang and L. O. Chua, "Impulsive stabilization for control and synchronization of chaotic systems: theory and application to secure communication," IEEE Transactions on Circuits and Systems I: Fundamental Theory and Applications, vol. 44, no. 10, pp. 976-988, 1997.

[6] C. Li, X. Liao, and K.-W. Wong, "Chaotic lag synchronization of coupled time-delayed systems and its applications in secure communication," Physica D: Nonlinear Phenomena, vol. 194, no. 3-4, pp. 187-202, 2004. 
[7] J. Meng and X.-Y. Wang, "Robust anti-synchronization of a class of delayed chaotic neural networks," Chaos, vol. 17, no. 2, Article ID 023113, 2007.

[8] X. Li and J. Cao, "Adaptive synchronization for delayed neural networks with stochastic perturbation," Journal of the Franklin Institute, vol. 345, no. 7, pp. 779-791, 2008.

[9] Q. Zhu and J. Cao, "Adaptive synchronization under almost every initial data for stochastic neural networks with timevarying delays and distributed delays," Communications in Nonlinear Science and Numerical Simulation, vol. 16, no. 4, pp. 2139-2159, 2011.

[10] H. Zhang, Y. Xie, Z. Wang, and C. Zheng, "Adaptive synchronization between two different chaotic neural networks with time delay," IEEE Transactions on Neural Networks, vol. 18, no. 6, pp. 1841-1845, 2007.

[11] S. C. Jeong, D. H. Ji, J. H. Park, and S. C. Won, "Adaptive synchronization for uncertain chaotic neural networks with mixed time delays using fuzzy disturbance observer," Applied Mathematics and Computation, vol. 219, no. 11, pp. 5984-5995, 2013.

[12] Y. Tang, R. Qiu, J. Fang, Q. Miao, and M. Xia, "Adaptive lag synchronization in unknown stochastic chaotic neural networks with discrete and distributed time-varying delays," Physics Letters A: General, Atomic and Solid State Physics, vol. 372, no. 24, pp. 4425-4433, 2008.

[13] Q. Zhu and J. Cao, "Adaptive synchronization of chaotic CohenCrossberg neural networks with mixed time delays," Nonlinear Dynamics, vol. 61, no. 3, pp. 517-534, 2010.

[14] D. Zhang and J. Xu, "Projective synchronization of different chaotic time-delayed neural networks based on integral sliding mode controller," Applied Mathematics and Computation, vol. 217, no. 1, pp. 164-174, 2010.

[15] V. I. Utkin, Sliding Modes in Control and Optimization, Springer, Berlin, Germany, 2001.

[16] J. F. Heagy, T. L. Carroll, and L. M. Pecora, "Experimental and numerical evidence for riddled basins in coupled chaotic systems," Physical Review Letters, vol. 73, no. 26, pp. 3528-3531, 1994.

[17] Y. Sun, J. Cao, and Z. Wang, "Exponential synchronization of stochastic perturbed chaotic delayed neural networks," Neurocomputing, vol. 70, no. 13-15, pp. 2477-2485, 2007.

[18] J. H. Park, "Synchronization of cellular neural networks of neutral type via dynamic feedback controller," Chaos, Solitons and Fractals, vol. 42, no. 3, pp. 1299-1304, 2009.

[19] X. Li and X. Fu, "Synchronization of chaotic delayed neural networks with impulsive and stochastic perturbations," Communications in Nonlinear Science and Numerical Simulation, vol. 16, no. 2, pp. 885-894, 2011.

[20] F. Ren and J. Cao, "Anti-synchronization of stochastic perturbed delayed chaotic neural networks," Neural Computing and Applications, vol. 18, no. 5, pp. 515-521, 2009.

[21] G. Zhang, Y. Shen, and L. Wang, "Global anti-synchronization of a class of chaotic memristive neural networks with timevarying delays," Neural Networks, vol. 46, pp. 1-8, 2013.

[22] T. Ma and J. Fu, "On the exponential synchronization of stochastic impulsive chaotic delayed neural networks," Neurocomputing, vol. 74, no. 5, pp. 857-862, 2011.

[23] H. Zhang, T. Ma, G.-B. Huang, and Z. Wang, "Robust global exponential synchronization of uncertain chaotic delayed neural networks via dual-stage impulsive control," IEEE Transactions on Systems, Man, and Cybernetics B: Cybernetics, vol. 40, no. 3, pp. 831-844, 2010.
[24] X. Li and R. Rakkiyappan, "Impulsive controller design for exponential synchronization of chaotic neural networks with mixed delays," Communications in Nonlinear Science and Numerical Simulation, vol. 18, no. 6, pp. 1515-1523, 2013.

[25] H. Zhang, T. Ma, J. Fu, and S.-C. Tong, "Global impulsive exponential synchronization of stochastic perturbed chaotic delayed neural networks," Chinese Physics B, vol. 18, no. 9, pp. 3742-3750, 2009.

[26] Q. Gan, "Synchronization of unknown chaotic neural networks with stochastic perturbation and time delay in the leakage term based on adaptive control and parameter identification," Neural Computing and Applications, vol. 22, no. 6, pp. 1095-1104, 2013.

[27] Q. Gan and Y. Liang, "Synchronization of chaotic neural networks with time delay in the leakage term and parametric uncertainties based on sampled-data control," Journal of the Franklin Institute, vol. 349, no. 6, pp. 1955-1971, 2012.

[28] Q. Gan, "Synchronisation of chaotic neural networks with unknown parameters and random time-varying delays based on adaptive sampled-data control and parameter identification," IET Control Theory and Applications, vol. 6, no. 10, pp. 15081515, 2012.

[29] Y. Tang, J. Fang, and Q. Miao, “On the exponential synchronization of stochastic jumping chaotic neural networks with mixed delays and sector-bounded non-linearities," Neurocomputing, vol. 72, no. 7-9, pp. 1694-1701, 2009.

[30] X. Li, C. Ding, and Q. Zhu, "Synchronization of stochastic perturbed chaotic neural networks with mixed delays," Journal of the Franklin Institute, vol. 347, no. 7, pp. 1266-1280, 2010.

[31] X. Mao, "A note on the LaSalle-type theorems for stochastic differential delay equations," Journal of Mathematical Analysis and Applications, vol. 268, no. 1, pp. 125-142, 2002.

[32] H. K. Khalil, Nonlinear Systems, Prentice-Hall, Upper Saddle River, NJ, USA, 1996.

[33] X. Yang, Q. Zhu, and C. Huang, "Lag stochastic synchronization of chaotic mixed time-delayed neural networks with uncertain parameters or perturbations," Neurocomputing, vol. 74, no. 10, pp. 1617-1625, 2011.

[34] Y. Sun and J. Cao, "Adaptive lag synchronization of unknown chaotic delayed neural networks with noise perturbation," Physics Letters A: General, Atomic and Solid State Physics, vol. 364, no. 3-4, pp. 277-285, 2007.

[35] B. Ksendal, Stochastic Differential Equations an Introduction with Applications, Springer, New York, NY, USA, 2005.

[36] Y. Wang, L. Xie, and C. E. de Souza, "Robust control of a class of uncertain nonlinear systems," Systems and Control Letters, vol. 19, no. 2, pp. 139-149, 1992.

[37] S. Boyd, L. El Ghaoui, E. Feron, and V. Balakrishnan, Linear Matrix Inequalities in System and Control Theory, SIAM, Philadelphia, Pa, USA, 1994.

[38] D. J. Higham, "An algorithmic introduction to numerical simulation of stochastic differential equations," SIAM Review, vol. 43, no. 3, pp. 525-546, 2001.

[39] M. Gilli, "Strange attractors in delayed cellular neural networks," IEEE Transactions on Circuits and Systems I: Fundamental Theory and Applications, vol. 40, no. 11, pp. 849-853, 1993.

[40] A. Pikovsky, M. Rosenblum, and J. Kurths, Synchronization: A Universal Concept in Nonlinear Sciences, Cambridge University Press, 2001.

[41] A. Arenas, A. Díaz-Guilera, J. Kurths, Y. Moreno, and C. Zhou, "Synchronization in complex networks," Physics Reports, vol. 469, no. 3, pp. 93-153, 2008. 
[42] W. Zhang, Y. Tang, Q. Miao, and W. Du, "Exponential synchronization of coupled switched neural networks with modedependent impulsive effects," IEEE Transactions on Neural Networks and Learning Systems, vol. 24, no. 8, pp. 1316-1326, 2013.

[43] W. Zhang, Y. Tang, X. Wu, and J. Fang, "Synchronization of nonlinear dynamical networks with heterogeneous impulses," IEEE Transactions on Circuits and Systems I: Fundamental Theory and Applications, vol. 61, no. 4, pp. 1220-1228, 2014.

[44] Y. Tang, H. Gao, W. Zou, and J. Kurths, "Distributed synchronization in networks of agent systems with nonlinearities and random switchings," IEEE Transactions on Systems, Man, and Cybernetics B: Cybernetics, vol. 43, no. 1, pp. 358-370, 2013. 


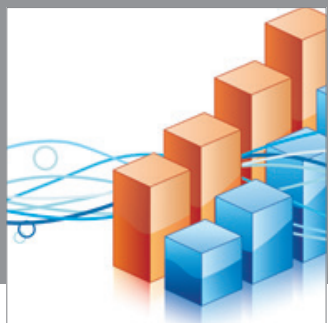

Advances in

Operations Research

mansans

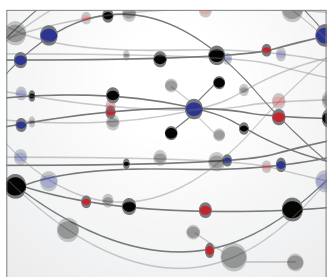

The Scientific World Journal
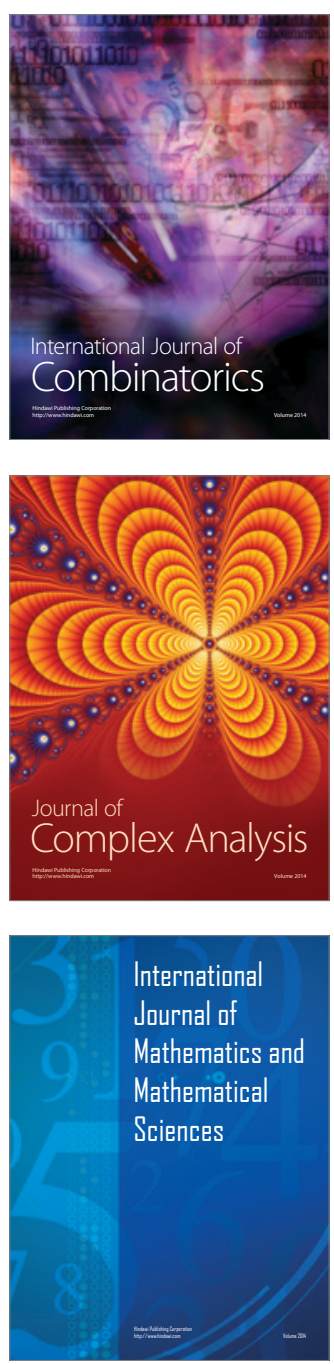
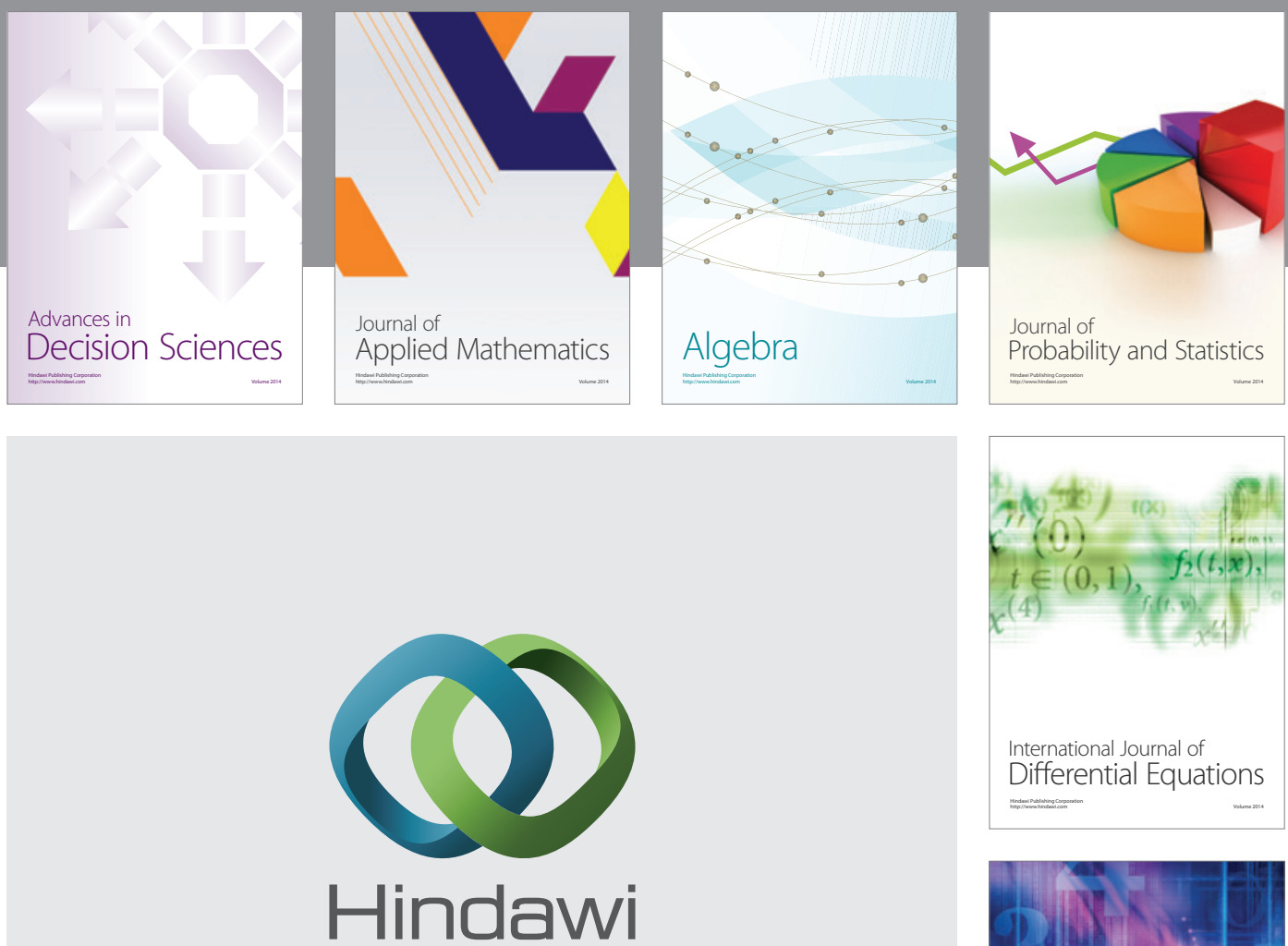

Submit your manuscripts at http://www.hindawi.com
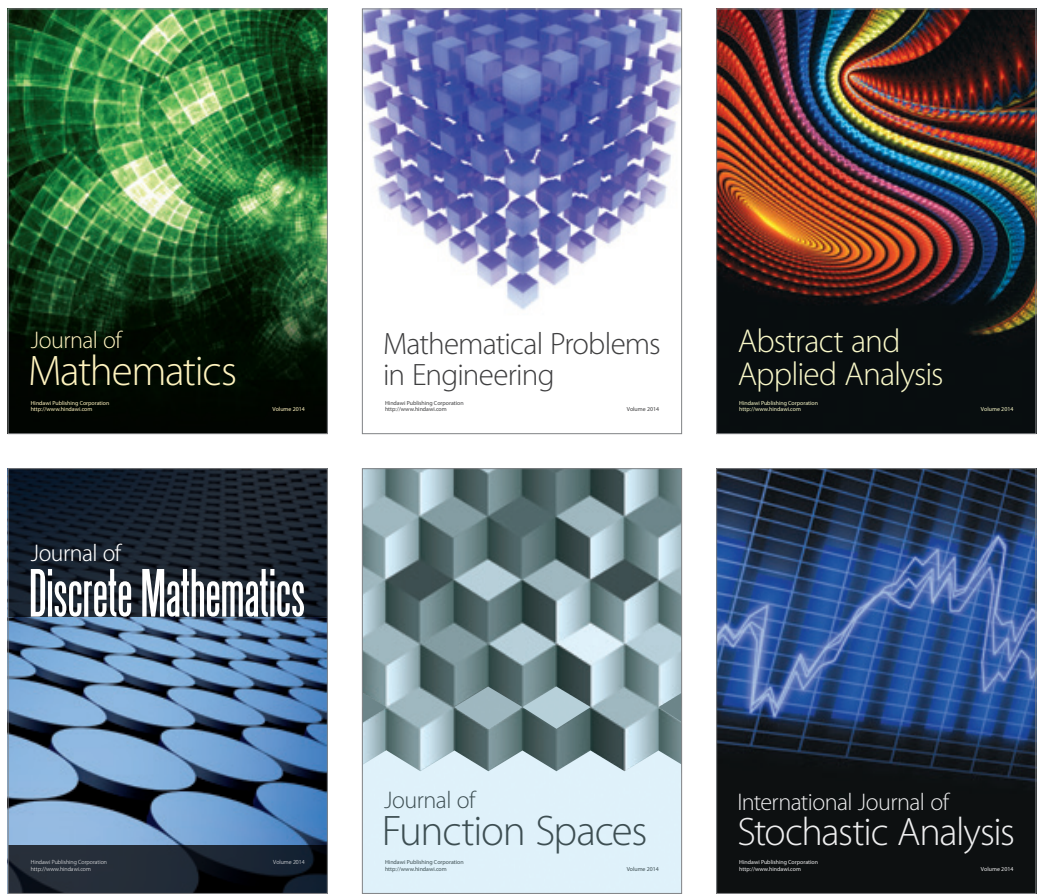

Journal of

Function Spaces

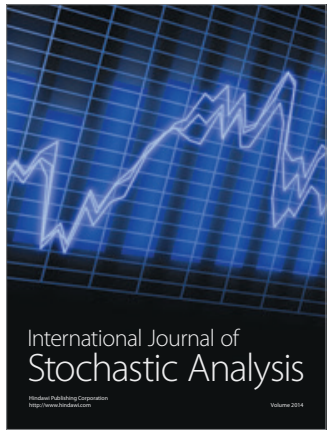

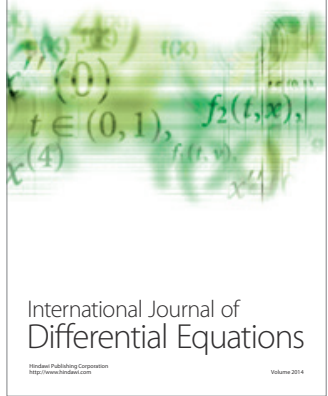
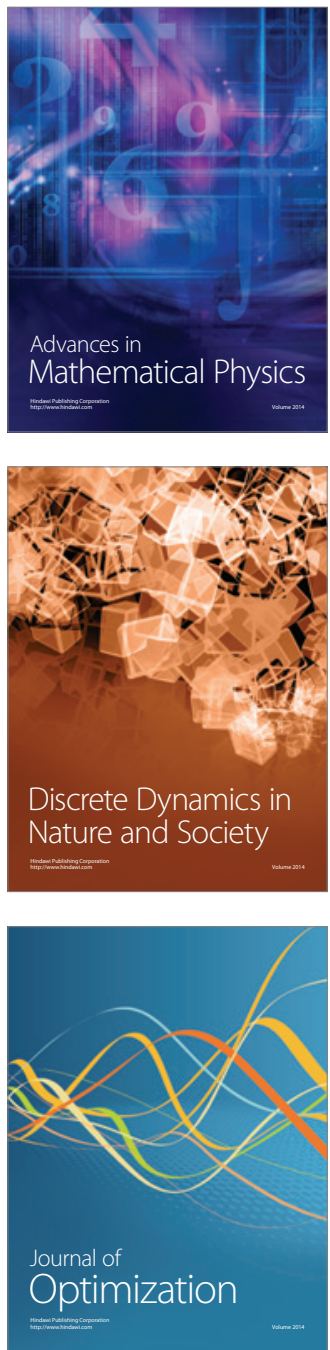